\title{
Strategies Used by Families to Cope with Chronic \\ Mental Illnesses: Psychometric Properties of the Family Crisis Oriented Personal Evaluation Scale
}

Ayşe SARI, ${ }^{1}$ Zekiye ÇETINKAYA DUMAN ${ }^{2}$

${ }^{1}$ Dokuz Eylül University, Institute of Health Sciences, Faculty of Nursing, Department of Psychiatric Nursing, İzmir, TURKEY ${ }^{2}$ Dokuz Eylül University, Faculty of Nursing, Department of Psychiatric Nursing, İzmir, TURKEY

Purpose: This study was aimed at investigating the psychometric properties of the Family Crisis Oriented Personal Evaluation Scale (F-COPES) for Turkish society, which assesses the coping skills of caregivers of individuals with chronic mental illnesses.

Design and Methods: The study was conducted with 153 family caregivers in the inpatient and outpatient units and İzmir Schizophrenia Solidarity Association. For the language validity, translation-back translation method was performed. For the content validity, expert opinions were obtained, for the construct validity, exploratory and confirmatory factor analysis was performed. For the reliability analysis, Cronbach alpha reliability coefficient was calculated and the test-retest reliability analysis was performed.

\section{Findings:}

* The content validity index of the scale was 0.96 .

* Factor loadings of the subscales ranged between 0.32 and 0.70 for the acquiring social support subscale, between 0.44 and 0.74 for the reframing subscale, between 0.54 and 0.74 for the seeking spiritual support subscale, and between 0.33 and 0.70 for the mobilizing to acquire and accept help subscale (Figure 1). The model fit indexes were as follows: $X^{2}$ $=327.269, \mathrm{df}=201, \mathrm{X}^{2} / \mathrm{df}=1.62, \mathrm{RMSA}=0.064, \mathrm{CFI}=$ $0.84, \mathrm{IFI}=0.84, \mathrm{GFI}=0.84$ (Table 1$)$.

* The Cronbach's alpha reliability coefficient for the overall scale was 0.82 (Figure 2).

Practice Implications: The results of the present study show that the levels of psychometric properties of F-COPES in Turkish society are acceptable. It is thought that it would be useful to use the F-COPES in the assessment of coping behaviors of individuals who give care to patients with a chronic mental illness and in studies to be conducted with caregivers of patients with chronic mental illness. It is also recommended to develop scales aimed at assessing families' strategies to cope with the chronic mental illness in Turkish culture

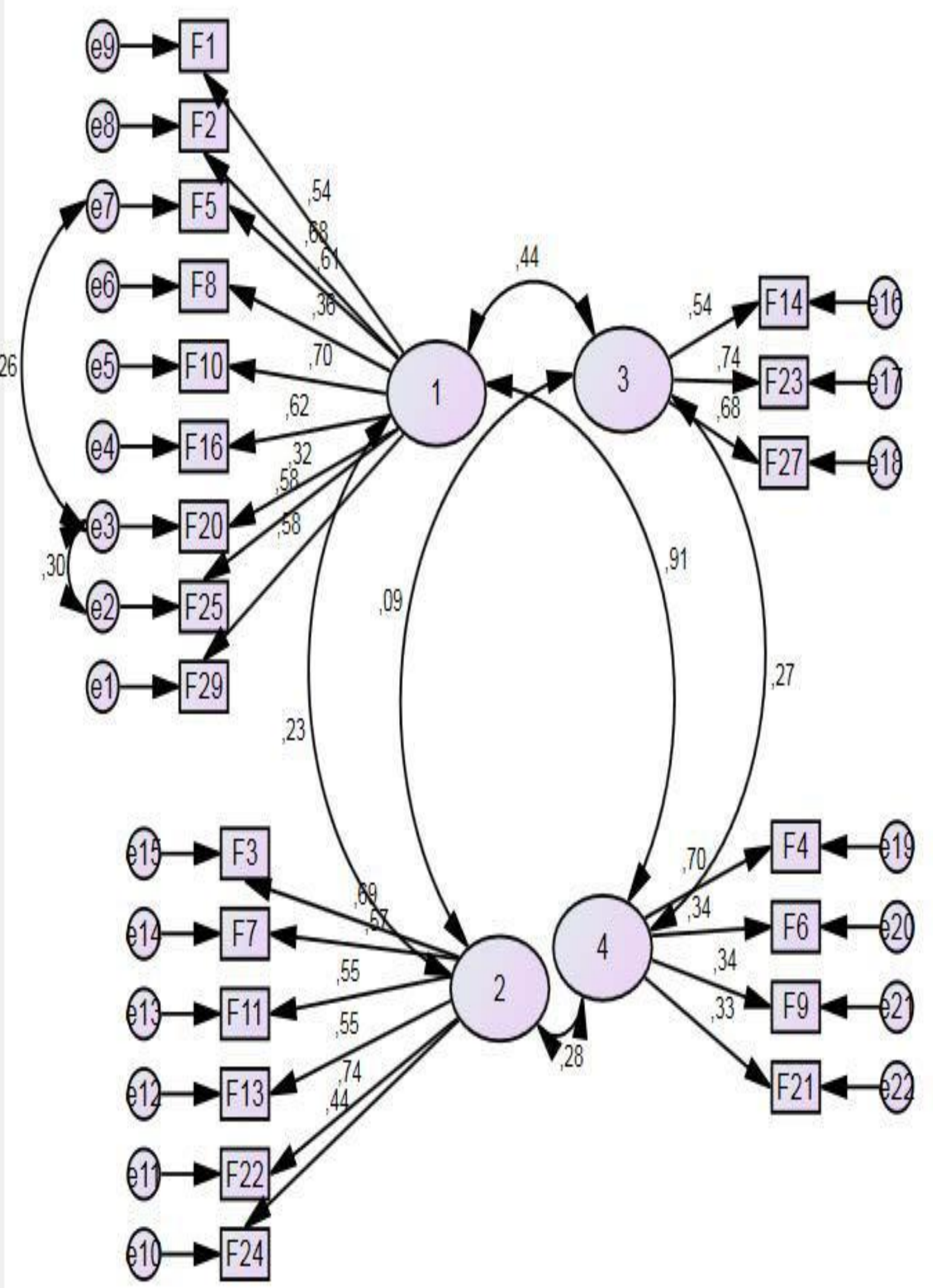

Figure 1. Confirmatory Factor Analysis of the F-COPES

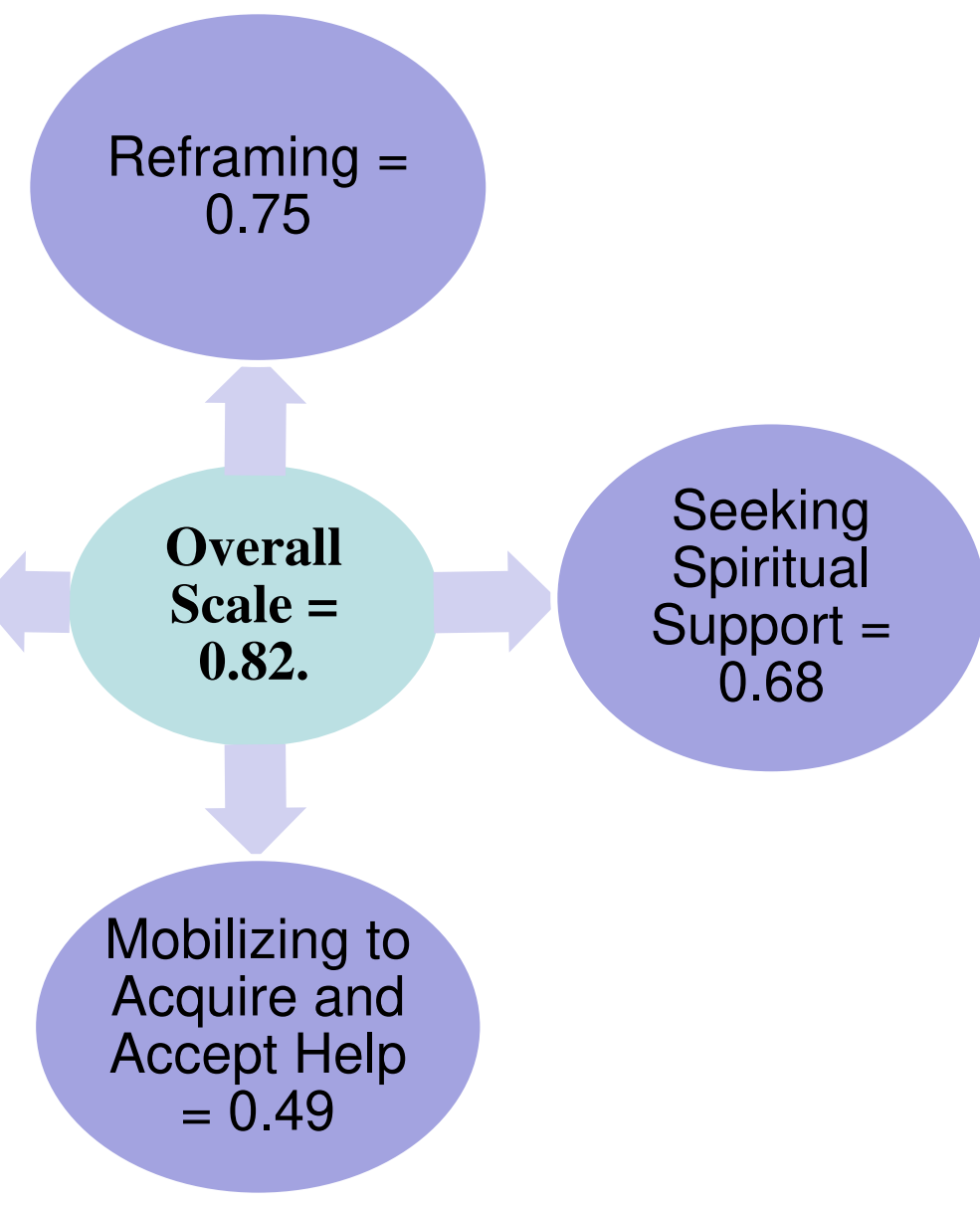

Figure 2. Cronbach's Alpha Coefficients of the F-COPES

Table 1. Model Fit Indices of of the F-COPES

\begin{tabular}{|c|c|c|c|c|c|c|c|c|}
\hline $\mathbf{X}^{2}$ & $\mathbf{D f}$ & $\mathbf{P}$ & $\mathbf{X}^{\mathbf{2}} \mathbf{d f}$ & \multicolumn{1}{|c|}{ RMSEA } & GFI & CFI & IFI & Hoelter $(\mathbf{p}=\mathbf{0 . 0 1})$ \\
\hline 327.269 & 201 & 0.00 & 1.62 & 0.064 & 0.84 & 0.84 & 0.84 & 117 \\
\hline
\end{tabular}

References:

- Beavers, A. S., Lounsbury, J. W., Richards, J. K., Huck, S. W., Skolits, G. J., \& Esquivel, S. L. (2013). Practical considerations for using exploratory factor analysis in educational research. Practical Assessment, Research \& Evaluation, 18.

McCubbin, H., \& Patterson, J. (1983). The family stress process; The double ABCX model of adjustment and adaptation. Marriage and Family Review, 6,7-37.

Lavee, Y., McCubbin, H. I., \& Patterson, J. M. (1985). The double ABCX model of family stress and adaptation: An empirical test by analysis of structural equations with latent variables. Journal of Marriage and the Family, 811-825. 